\title{
Protée
}

\section{Chassés-croisés à propos du film Dancing (Patrick Mario Bernard, Xavier Brillat et Pierre Trividic - France, 2003)}

\section{Marie-Françoise Grange}

Volume 37, numéro 3, hiver 2009

Regards croisés sur les images scientifiques

URI : https://id.erudit.org/iderudit/038811ar

DOI : https://doi.org/10.7202/038811ar

Aller au sommaire du numéro

Éditeur(s)

Département des arts et lettres - Université du Québec à Chicoutimi

ISSN

0300-3523 (imprimé)

1708-2307 (numérique)

Découvrir la revue

Citer cet article

Grange, M.-F. (2009). Chassés-croisés à propos du film Dancing (Patrick Mario Bernard, Xavier Brillat et Pierre Trividic - France, 2003). Protée, 37(3), 119-124. https://doi.org/10.7202/038811ar
Résumé de l'article

Le présent article étudie dans le film Dancing les enjeux de la question du genre, genre filmique et genre identitaire. Ainsi, le début du film refuse de mettre en place clairement des consignes de lecture ; le personnage de l'Idiot " dégenré " performe l'image autoportraitique dont il énonce les limites et frontières. 


\section{CHASSÉS-CROISÉS À PROPOS DU FILM DANCING \\ (Patrick Mario Bernard, Xavier Brillat et Pierre Trividic - France, 2003)}

MARIE-FrANÇOISE GRANGE

Dancing ${ }^{1}$ croise deux registres a priori très distincts : d'une part, la représentation de l'homosexualité à l'écran; d'autre part, l'autoportrait tel que le cinéma peut le mettre en scène à la suite de la peinture et des autres arts plastiques. Certes, Dancing n'est pas une exception en la matière, nombre de films, qu'ils soient expérimentaux (par exemple, Ixe de Lionel Soukaz - France, 1980), qu'ils appartiennent au cinéma documentaire (cas de La pudeur ou l'impudeur de Hervé Guibert - France, 1991) ou au cinéma fictionnel (comme le tout récent J'ai tué ma mère de Xavier Dolan - Canada, 2009), imbriquent à des degrés divers ces deux composantes. Tout film proposant l'autoreprésentation de son réalisateur, par ailleurs homosexuel, mêle directement ou indirectement ces deux problématiques dont l'une, I'homosexualité, relève de la représentation des identités sexuelles et I'autre, I'autoportrait, d'une question d'esthétique du cinéma. Ce qui caractérise Dancing est donc non pas cette imbrication homosexualité/autoportrait, mais la manière dont sont questionnées et remises en cause, à travers ces deux registres, les catégories: catégories de genres constitutives de l'identité individuelle, catégories de genres cinématographiques dans lesquelles s'élaborent des types filmiques. C'est à travers un ballet étrange interprété par des personnages, pris dans des effets de ressemblance plus ou moins décalés, que Dancing ébranle les frontières et interroge les limites à partir desquelles se structurent les comportements et les habitudes de penser. Dans l'intrigue, la figure du double, les effets de miroir et autres variations déplacent les identités et ouvrent sur des possibles multiples faisant cohabiter différents personnages parfois surprenants, comme celui de I'Idiot « dégenré». Quant au film lui-même, glissant d'autoreprésentations en autoportraits sans véritablement les affirmer en tant que tels, il est une sorte d'objet non identifiable qui bouscule les consignes de lecture et perturbe les repères.

\section{Personnages et autoreprésentation}

Dancing est un film narratif représentatif qui se construit autour d'une intrigue avec personnages. Bien qu'un de ses principaux personnages soit plasticien, ce film n'est pas un film d'artiste voué à une recherche plastique audiovisuelle, telles les œuvres cinématographiques de Soukaz, dont lxe mentionné plus haut. Avec Dancing, nous avons bel et bien affaire à un film de type fictionnel, qui fut, d'ailleurs, lors de sa sortie en salle en France, distribué par un grand circuit (UGC).

Les deux protagonistes du film forment un couple: ils vivent ensemble, en Bretagne, au bord de la mer. L'un, Patrick, est scénariste, l'autre, René, est plasticien. Le premier participe à des interviews, il est amené à se déplacer à plusieurs reprises (Copenhague, Paris). Le second prépare une exposition, reste le plus souvent à la maison ou à proximité (il se promène sur la plage, fait les courses), passe du temps dans son atelier et communique, par le biais du réseau Internet, avec son galeriste. Les deux hommes (René et Patrick) partagent le quotidien, ont une relation harmonieuse, sans conflits ni éclats: ce sont deux homosexuels qui vivent sans problème particulier et sans détour leur sexualité, et dont l'orientation ne semble l'objet d'aucune difficulté. Ils sont, séparément et ensemble, socialement intégrés au point de ne pas soulever la question de leur intégration: cette question n'est jamais effleurée et encore moins posée. Leur homosexualité est une évidence qui n'est plus à interroger, elle est là comme pratique sexuelle, comme mode de vie, comme manière de penser et d'être dans le monde. Le propos diégétique 
n'est donc pas organisé autour d'une revendication militante, comme peut l'être notamment celui du film Harvey Milk de Gus Van Sant (États-Unis, 2009).

Premier intérêt de Dancing: deux homosexuels vivent harmonieusement leur orientation; ouverts sur le monde, ils évoluent dans un milieu, il est vrai, protégé, dans lequel ils se sentent plutôt bien. Second intérêt du film : deux des trois réalisateurs de Dancing (Pierre Trividic et Patrick Mario Bernard) interprètent les deux rôles principaux. Si l'on ne peut parler à ce stade d'autoportrait ${ }^{2}$, peut-être peut-on déjà, dans un premier temps, remarquer l'autoreprésentation ${ }^{3}$ de deux des réalisateurs, apparaissant dans leur propre film.

Ce dispositif de mise en scène, centré sur une double autoreprésentation (ce qui est très rare), tend largement vers l'autofiction ${ }^{4}$, au journal filmé, au portrait de couple, le tout doublé d'une sorte d'autoportrait(s)... à deux.

J'ai tendance à penser que ce film ne ressemble à rien, au sens positif de l'expression bien sûr. II ne ressemble à rien et son intérêt réside justement dans cette non-ressemblance qui le fait sortir des sentiers battus ${ }^{5}$.

Le fait que les personnages soient homosexuels ne peut être tenu pour raison de ce caractère atypique du film. Être homosexuel, d'une part, n'a rien d'exceptionnel, d'autre part, cela ne confère pas un passeport pour voyager sur les terres de I'originalité. En revanche, la liberté autant que l'assurance de ton tiennent peut-être à la capacité d'accepter et d'exploiter la notion même de différence qui, par définition, place toujours à côté et, de fait, déplace les points de vue ${ }^{6}$.

\section{Début du film et contrat de lecture}

Le genre cinématographique auquel appartient Dancing est relativement indéterminable, notamment son début. Si, en général, les premiers plans de films installent le spectateur face à une ambiance, un genre cinématographique, une atmosphère, et posent d'emblée les enjeux ${ }^{7}$ qui seront exploités dans le reste du film, Dancing joue son entrée en matière tout autrement.

Le début de Dancing est très ambigu: plusieurs gros plans morcellent un paysage (herbes, eau), un corps (une main, un crâne chauve, un pied). Le montage n'élabore pas d'unité globale à partir de ces morceaux successifs. Les angles de prises de vues rendent difficiles les points de repère et les ancrages spatiaux et narratifs: où sommes-nous? Quel(s) personnage(s)? Quel type d'action? Il faudra, par exemple, attendre la septième minute du film pour que nous soit montré le visage d'un des personnages (René), alors que c'est essentiellement lui que la caméra a suivi jusque-là. II sera très difficile de saisir ce que les personnages font. C'est seulement, là aussi, au bout de plusieurs minutes que le spectateur commencera à comprendre l'organisation des plans entre eux et déchiffrera par bribes le comportement de René, à l'extérieur, et de Patrick, à l'intérieur ou au seuil de la maison ${ }^{8}$. Le premier se promène sur la plage, joue avec un ourson en plâtre, le trempe dans l'eau, prend des photos, reste allongé dans l'herbe; le second guette l'arrivée de quelqu'un. Tout cela met du temps à devenir explicite, l'ensemble est éclaté, le montage n'embraye pas directement sur une action, pas plus qu'il n'installe entre lui et son spectateur un contrat de lecture défini et précis.

Ainsi, certains plans pourraient apparenter Dancing à un film policier: le corps de René, allongé et découpé par les cadres successifs, semble inanimé, en attente d'être découvert par on ne sait quel passant; d'autres plans pourraient inscrire le film dans une démarche de type expérimental - les cadrages exposent leur focale et l'usage qui est fait du gros plan vise plus à cerner une distance qu'une réalité -; d'autres encore décomposent un monde, par vues juxtaposées les unes à la suite des autres, dans une perspective descriptive sans cohérence narrative établie.

Le début du film est hybride: il ne choisit pas et s'autorise à circuler d'un plan à l'autre sans lien évident, à prendre son temps. Il amène son spectateur à lâcher prise, à accepter la ballade au cœur d'un monde non encore organisé où tout et rien peuvent devenir possibles. Il faudra attendre pour que le propos s'affirme dans une direction donnée, mais toujours l'étrangeté guettera au détour de tel ou tel plan, laissant présager l'on ne sait trop quoi, laissant donc le spectateur s'approprier et gérer comme bon lui semble ces effets de surprise, la plupart du temps sans réponse ni explication.

Puis l'intrigue se déplace et s'installe. Le film nous propose de suivre le quotidien d'un plasticien, entre ses hésitations, ses angoisses et ses certitudes, le tout ponctué par le journal filmé qu'il tient sur Internet, en images, et qu'il adresse à son galeriste.

\section{De l'autoreprésentation à l'autoportrait}

Si nous recoupons ce work in progress de René, personnage du film, avec l'autoreprésentation d'un des réalisateurs (Patrick Mario Bernard, interprète de René), les effets d'échanges brouillent de nouveau le propos et relancent l'incertitude toujours aux aguets.

On le sait, Patrick Mario Bernard est plasticien, réalisateur de film et travaille avec son compagnon dans la vie, Pierre Trividic 
qui, lui, interprète Patrick dans le film, le compagnon de René. Ce savoir-là ne nous est pas communiqué directement par le film lui-même, mais la situation rejoint ici celle de tout autoportrait lorsque celui-ci ne porte pas un titre suffisamment explicite comme autoportrait ${ }^{9}$. En revanche, l'autoreprésentation des réalisateurs est affirmée par le générique de fin. L'autoreprésentation n'est pas équivalente à l'autoportrait ${ }^{10}$, en tout cas au cinéma, mais l'autoreprésentation peut servir d'assise à l'autoportrait. Quant au savoir sur les liens entre personnages et personnes, bien qu'extrafilmique, il fonctionne, en mineur ou en majeur, selon les spectateurs. En résumé, le personnage de René est de l'ordre de la fiction. Son interprétation par un des réalisateurs (Patrick Mario Bernard), par le biais donc d'une autoreprésentation, injecte dans la fiction une part de documentaire, elle tisse certains échos entre le personnage et la personne ouvrant à la confusion entre les niveaux narratifs. Les ressemblances plus ou moins serrées, plus ou moins lâches, entre vie du personnage et vie du réalisateur infléchissent le discours filmique vers le portrait d'un artiste par lui-même. L'autoreprésentation fait glisser le film de pure fiction à la possibilité de l'autoportrait.

Le portrait de René devient de manière sous-jacente celui de Patrick Mario Bernard; dès lors, celui de Patrick devient celui de Pierre Trividic; et le portrait du couple filmique, René et Patrick, devient celui du couple des réalisateurs.

En effet, les deux réalisateurs nous racontent quelque chose de leur vie commune, quelques-unes de leurs manières de voir le monde, de l'appréhender; ils se font les porte-parole directs (en s'autoreprésentant) de leur façon d'être et d'entendre, de voir et de vivre. Ils se transposent en René et Patrick pour mieux (ou moins? ou plus?) parler des images qui peuplent leurs univers de réalisateurs, de créateurs, d'artistes. Nous passons alors de l'autoreprésentation à l'autoportrait, et cela en glissant à travers une dimension autofictionnelle ${ }^{11}$. Restent les écarts entre une réalité, celle des réalisateurs, transposée dans un monde filmique, et un monde filmique, celui des personnages porteurs, à travers des résonances plus ou moins troublantes, d'une vérité documentaire.

Sous couvert de leur clone respectif, le couple dans la vie et dans le film, ou l'un des deux, s'autorisent quelques digressions dans lesquelles s'échangent non seulement des impressions, mais encore des mondes. Or, ces mondes se constituent comme parallèles. Y cohabitent plusieurs semblables, plusieurs vérités, plusieurs possibles. Ces mondes, en se croisant - car même les parallèles dans cet univers à géométrie variable peuvent se rencontrer ${ }^{12}-$, sont les seuls à même de dire quelque chose de la vérité: ni toute une, ni toute autre, mais une car autre, c'est-à-dire toujours différente.

N'est-ce pas le propre de l'autoportrait que de naviguer entre savoirs et ignorances, reflets et certitudes, même et autre? N'estce pas là que s'effleurent les images, se déplacent les regards? N'est-ce pas là que l'on se confronte à la fuite inéluctable du signifiant? Lorsque l'autoportrait est double et que tendent à se confondre les personnages, le mouvement s'amplifie et l'ensemble perd d'autant plus ses marques.

Des effets de miroir s'établissent entre les deux protagonistes unis par une vie commune et par des liens non seulement affectifs et sexuels, mais également morphologiques. Patrick et René se ressembleraient presque: les deux se portent bien et, comme pour beaucoup de couples, on les confond au téléphone. De plus, quand ils endossent leurs combinaisons de plongée pour aller nager, la caméra les tient sous un angle éloigné, de dos, et veille à ne plus permettre de les distinguer: deux silhouettes semblables partagent leur bain de mer. Le film joue de cette ressemblance. Que les deux hommes soient de type «nounours» souligne d'autant l'effet en question ${ }^{13}$.

Mais, qu'on ne s'y trompe pas, le problème n'est pas de portraiturer le couple selon les similitudes des deux partenaires. Il s'agit bien plutôt d'amorcer, par le phénomène de la ressemblance entre les deux hommes, les glissements de double à double. Il s'agit d'amorcer ou de reprendre la démultiplication qui s'empare de René lorsqu'il rencontre le personnage de l'Idiot et fait l'expérience de sa propre métamorphose en devenant lui-même l'Idiot.

\section{L'Idiot}

Qu'est-ce que ce personnage? L'Idiot arrive brusquement par la trappe de l'atelier qui communique avec les caves de la maison. Il est une figure burlesque ayant gardé non pas le tragique du burlesque, mais sa faculté à porter l'absurde jusqu'au comique.

L'Idiot, à proprement parler, surgit dans la vie de René comme un diable sortant de sa boîte. Il prend la tenue des Bernard Brothers, couple de comiques du music-hall anglo-saxon des années 1940. Le Bernard porte sur un corps nu une robe à bretelles, froncée à la taille qui lui arrive aux genoux, il arbore un superbe nœud qui lui encadre la tête. Sa position favorite est d'être accroupi. Il ramasse au sol des miettes ou des cailloux imaginaires, un à un. Lorsqu'il se redresse, il a tendance à effectuer de façon récurrente, et donc insistante, le même geste de la main: I'index et le pouce se touchent à plusieurs reprises. 
Le Bernard grimace, ne parle pas, il prononcera dans le film une seule phrase par la voix dédoublée de René. Le Bernard, si l'on en croit son modèle d'origine (les Bernard Brothers), est de sexe masculin et de genre féminin.

Que le Bernard du film apparaisse sous les traits de René, homme solide avec un certain embonpoint, grand, chauve, avec des bacchantes à la gauloise, renforce la drôlerie de la créature. Le comique ne provient pas du transformisme du personnage: un homme habillé en femme comme c'est le cas, par exemple, pour les deux personnages principaux de Certains I'aiment chaud de Billy Wilder (États-Unis, 1959). Le nouveau Bernard ne fonctionne pas à ce niveau-là ${ }^{14}$. Son comique vient de l'hybridité de la créature, revendiquée en tant que telle car surexploitée, et non de la mise en scène de la dualité du masculin et du féminin portée par l'image classique du travesti au cinéma (Michaud, 2000: 86). C'est d'ailleurs en cela que le burlesque, qui est exploité par la répétition systématisée, le travestissement du corps, l'absurdité des situations, dérape. Avec le Bernard réinterprété, le comique devient beaucoup plus décapant et ravageur, car dénonciateur de valeurs sous-jacentes insidieusement à l'œuvre dans I'ordre social des identités. En cela, le Bernard amuse et dérange.

Le nouveau Bernard mélange et donc bouscule allègrement les catégories, pas seulement de genres, en les faisant cohabiter: il est vrai qu'il porte la moustache (signe de genre masculin), la robe (signe de genre féminin), mais encore se risque-t-il à faire cohabiter l'adulte de sexe mâle (la moustache), l'enfant (le jeu répétitif, la position accroupie), la petite fille (la robe avec bretelles et le nœud dans les cheveux), I'artiste autoreprésenté en créature déjantée, la proposition plastique (le Bernard est un portrait, voire un autoportrait - de René/de Patrick Mario Bernard -, et s'expose au regard de l'ami et du galeriste avant qu'il ne devienne objet d'art de galerie ou de film). Il est aussi l'incarnation de l'Idiot dont la fonction n'est que d'être là, dans le présent de sa présence. Le Bernard, comme l'Idiot, est singulier, coupé du langage, il est seulement là. Il fait écran, il est incompréhensible car il se tient à une distance différente de celle de tout un chacun avec le réel. L'Idiot (voir Rosset, 1977) est étranger au monde, il est ailleurs. Il est déplacé et pose problème.

L'idiot en Bernard ose franchement l'hybridité, sans complexe et sans détour. Il devient I'Idiot par excellence, hors classification 15 , «le particulier», hors concept, hors catégorie et hors genre. Si l'Idiot est l'une des formes du Bernard, le Bernard, quant à lui, vaut comme paradigme de l'Idiot.
Ainsi, le Bernard propose une nouvelle race d'Idiot et en compose une nouvelle figure. II n'est pas seulement celui qui est singulier, à part ${ }^{16}$, il n'est pas seulement, non plus, celui qui n'est pas doté de langage, qui ne comprend rien étant situé en dehors de tout processus de communication, celui dont l'attitude est étrange, jamais connue par avance, mais plus encore, il est ici «dégenré »17. En cela, il prend de la distance par rapport à la plupart des personnages d'idiots. Car le Bernard explore l'ailleurs, le redéfinit. Par une accumulation d'emblèmes qui le pose en désignateur de catégories, le Bernard ne s'apparente qu'en partie à ces catégories, autant dire qu'il ne s'y apparente plus du tout. En effet, la catégorie ne fonctionne que sur une valeur oppositionnelle, ainsi le masculin n'est pas du féminin, et la suppression de la notion même d'opposition ouvre des champs aux multiples attraits, atours (voir la parure du Bernard), aux identités multiples et autres manipulations. Venu d'ailleurs, d'un autre monde, dans lequel les règles peuvent être plus facilement repensées, ou du moins désignées, le Bernard déplace l'Idiot car il réinvente les territoires de l'ailleurs. Désormais, le Bernard nouveau reprend le flambeau de la quête autoportraitique.

Si le corps est une réalité bien présente dans le film (voir les scènes relatives aux rapports sexuels entre les deux hommes, la préparation du corps avant la relation sexuelle), I'Idiot, quant à lui, soulève la question de l'identité dans laquelle prend place celle du genre. Par le Bernard, par cette drôle de créature, l'autoportrait se saisit de l'identité et l'inscrit aux confins du réel, dans I'«ici» et «maintenant». L'autoportrait en Idiot/I'Idiot comme autoportrait 18 « ne trouvera [...] rien d'autre que ce qu' [il] y cherchait réellement: c'est-à-dire, précisément, rien » (Rosset, 1977: 153) ${ }^{19}$. En ce sens, I'autoportrait, au travers de ce nouvel Idiot, porte la quête identitaire aux limites de sa signifiance, aux limites de ses possibilités, et trouve là un territoire ouvert sur l'étranger. L'autoportrait révèle alors ses propres impossibilités: il est ce rien du réel ici et maintenant. Comme tout image, il ne peut que rater son but.

Dancing se déplace de double en double, de portrait en autoportrait, d'autoportrait en autofiction. Il fait voler les frontières, bouscule les limites : René (personnage)/Patrick Mario (réalisateur) est un Bernard. Il leur arrive à tous deux (tous trois?) de partager le même repas à la même table, de prendre place dans le même espace, face à face, dans l'atelier, et de se regarder. Lorsque le Bernard articule la seule phrase qu'il prononce, il le fait par la voix dédoublée de René/Patrick Mario et lorsque René/ Patrick Mario s'adresse à son Bernard, il l'interpelle avec 
une voix travaillée en écho, résonante. La recherche de l'identité par le biais du portrait de l'autre ou de l'autoportrait (ce qui reviendrait au même) dérive vers un double greffé sur l'unité ainsi déconstruite, un double qui ne remplace pas, mais qui cohabite, en supplément, pour reprendre une terminologie derridienne. Le Bernard est la dernière proposition plastique de René. Entre ours de différentes tailles, autres images de lui-même, cette chose hors concept, hors catégorie, hors genre, hors tout servira à son tour de portrait/autoportrait, elle ne mettra pas un terme à la recherche de la bonne image, mais inscrira l'impossible lieu de cette image «toute».

Le portrait de l'artiste en Bernard devient la métaphore de I'impossible autoportrait identitaire lorsque celui-ci se veut être pensé à partir de critères exclusifs. Il est déconstruction de cette pensée de l'identité. À ce titre, il devient l'autoportrait par excellence, c'est-à-dire la signifiance et l'insignifiance conjuguées de l'image de soi, le tout et le rien de cette image, le sujet/objet insaisissable; il est la perte de soi par soi, sans limites et sans fin, il est ce «rien» du réel dont nous entretient Clément Rosset.

\section{Conclusion}

Le Bernard communique sa force disruptive, son énergie venue de contrées étrangères, il laisse sur son passage un goût que seuls certains sont à même de connaître ou de reconnaître. Le galeriste de René, invité des deux protagonistes, passera une nuit bien étrange: de la cave, deux, trois, cinq, sept, dix galeristes, tous semblables, sortiront les uns après les autres. Ils investiront I'atelier de René et accompliront, en combinaisons de plongée, diverses galipettes, et autres figures tout aussi surprenantes, sous les yeux ébahis de leur référent, le supposé vrai galeriste.

Le Bernard est une figure explosive au sens propre comme au sens figuré. Sa dimension farcesque, fantastique, non normée, déstabilise les repères. Venu d'ailleurs, insaisissable, il produit des figures, des semblants, des identités pensées sous la coupe du multiple. Il détruit les catégories, car il se moque des logiques oppositionnelles. Le Bernard produit un sujet plus qu'il ne le transcrit. C'est en cela qu'il est performance autoportraitique.

\section{NOTES}

1. Film français de Patrick Mario Bernard, Xavier Brillat et Pierre Trividic (35 mm, 1 h 33, 2003).

2. L'autoportrait est un portrait de soi par soi.

3. J'entends par le terme d'autoreprésentation la mise en scène par le réalisateur de son propre corps à l'intérieur de son film. Qu'il joue son propre rôle ou qu'il en interprète un autre n'est pas définitoire de I'autoreprésentation.

4. Serge Doubrovsky définit ainsi le terme d'autofiction en littérature: «L'autofiction, c'est la fiction que j'ai décidé, en tant qu'écrivain, de me donner de moi-même et par moi-même, en y incorporant, au sens plein du terme, l'expérience de l'analyse non point seulement dans la thématique mais dans la production du texte» (1988: 77). La question de l'autofiction est d'autant plus pertinente que le film se présente comme un film de fiction.

5. J. Y. Jouhannais (2003) voit, dans ce film, un film idiot. Il le distingue du film de Lars Von Trier, Les Idiots (Danemark, 1998), film sur l'idiotie, alors que Dancing pousse les choses plus loin en proposant un film lui-même idiot.

6. Point de vue est utilisé ici au sens de lieu à partir duquel on regarde. 7. «[...] les premiers plans d'un film revêtent une importance toute particulière: opérant la transition entre notre monde (l'espace de la salle de projection) et le monde du film (la diégèse), ils ont pour fonction de donner au spectateur les consignes de lecture qui lui permettront d'adopter un mode de production de sens et d'affects adapté » (Odin, 2000: 76).

8. La musique du film joue un rôle essentiel dans cette étrangeté du film. Plutôt intrigante, elle renforce l'indécision quant au genre filmique, le tirant vers une tonalité suspensive, car trop fortement pesante sur des images qui sont, la plupart du temps, plus légères. Cette musique, souvent en décalage avec les images, ne renforce pas une catégorie précise de genre cinématographique. En ce sens, elle prend une part active dans la déconstruction du genre filmique.

9. Autoportrait en titre de tableau instaure un contrat de lecture: la personne représentée sur la toile est censée avoir les traits de l'artiste. Au cinéma, il en est de même. De plus, l'autoportrait reprend le contrat de lecture de l'autobiographie (Grange, 2008: 63), tel que P. Lejeune (1975) I'a décrit à propos de la littérature, par laquelle l'auteur, le narrateur et le personnage sont une même et unique personne.

10. Si I'autoportrait est un portrait de soi par soi, I'autoreprésentation ne fait pas nécessairement portrait. L'autoportrait ouvre sur la question du Sujet; I'autoreprésentation, quant à elle, ouvre sur la question de la représentation du corps de l'artiste au sein de son œuvre. Voir, sur cette distinction, M.-F. Grange (2008: 61-65).

11. Le film ne se situe pas dans une problématique documentaire, si ce n'est par le simple fait que les personnages ne portent pas les mêmes noms que les réalisateurs. Aussi, ces derniers n'interprètent pas directement leur propre rôle, mais tiennent un rôle autre que le leur. L'autoreprésentation (mise en scène du corps des réal isateurs), aux échos d'autoportrait (construction d'un portrait de soi par soi), passe donc par le biais de l'autofiction (construction d'une fiction pour élaborer une vérité sur soi). À chaque niveau - autoreprésentation/autoportrait/ autofiction - correspond un type de contrat de lecture.

12. C'est en substance ce que le scientifique danois explique aux deux personnages dans le film.

13. Les effets de miroir entre personnages ont pu être exploités, notamment dans le passé, par des films comme Persona de Ingmar Bergman (Suède, 1966) : la relation ambiguë entre Alma (I'infirmière) et Elisabeth 
(I'actrice) s'élabore sur ces effets qui mènent au trouble et à la confusion des personnalités entre les deux femmes. Dans Dancing, la question du double et de la ressemblance ne cherche pas à construire une quelconque ambiguïté ou déstabilisation psychologiques entre les protagonistes. II s'agit ici de jouer, au sens ludique du terme, sur des visibilités qui se renvoient la balle, cela dans un très grand respect et une très grande complicité.

14. Si les personnages de Billy Wilder sont bien des créatures de sexe masculin prenant, tant bien que mal, afin de se cacher, les atours du genre féminin, ils ne revendiquent pas le genre féminin comme élément de leur propre identité. Mais leur travestissement leur fait porter «ensemble le spectacle de la féminité et celui de la virilité contrariée » (c'est moi qui souligne), comme le remarque P.-A. Michaud (2000: 86) à propos du travesti au cinéma. En revanche, le Bernard, interprété par René, est de sexe masculin et revendique les genres masculin et féminin. Si les personnages de Billy Wilder miment de manière caricaturale la féminité (et en retour la masculinité), le Bernard, quant à lui, dénonce la caricature des constructions de genre et ne fonctionne pas sur une opposition "genrée».

15. Valérie Deshoulières, dans Métamorphoses de l'idiot (2005), note la difficulté des nosographies du XIXe pour classer l'idiot.

16. Premier sens du terme idiot (du grec idios): "simple, particulier». $\mathrm{C}^{\prime}$ est avec le terme latin, idiotes, que l'idiot prend une valeur négative. L'idiotes est l'inculte, sans instruction. De ces deux étymologies découlent les deux sens du terme idiot: simple, particulier et faible d'esprit. La plupart du temps, l'idiot est défini négativement, il est celui qui est privé d'intelligence, incapable de former des concepts (Deshoulières, 2005: 17-18)

17. «Dégenré », c'est-à-dire déstabilisant la catégorie du genre, conçu comme catégorie donnée, unitaire et oppositionnelle. Le Bernard interprète la catégorie du genre et lui restitue sa valeur performative. Judith Butler (2005) insiste tout particulièrement sur le caractère performatif du genre. Or, le Bernard semble bien lui restituer cet enjeu performatif. 18. II ne s'agit pas, bien évidemment, de penser l'idiot "dégenré » comme créature impossible, mais de penser l'image autoportraitique, quelle qu'elle soit, comme impossible. Or, sous la forme de l'Idiot, I'image autoportraitique devient l'étrangère par excellence, c'est-à-dire inaccessible comme entité, comme identité. L'autoportrait reprend alors place au cœur de sa propre performativité (Grange, 2008).

19. C'est ainsi que Clément Rosset termine son étude sur l'Idiot: « $[\ldots]$ ne cherchez pas le réel ailleurs qu'ici et maintenant, car il est ici et maintenant, seulement ici et maintenant. Mais, si l'on ne veut pas du réel, il est préférable, en effet, de regarder ailleurs: d'aller voir ce qui se passe sous le tapis, ou en Amérique du Sud, ou dans la mer des Caraïbes, n'importe où pourvu qu'on soit assuré de n'y jamais rien trouver. Car on n'y trouvera jamais rien d'autre que ce qu'on y cherchait réellement: c'est-à-dire, précisément, rien » (1977: 152-153).

\section{RÉFÉRENCES BIBLIOGRAPHIQUES}

BUTLER, J. [2005] : Trouble dans le genre, Paris, La Découverte. CAMPAN, V. et C. RANNOUX (dir.) [2005] : La Licorne: le journal aux frontières de l'art, Rennes, Presses universitaires de Rennes. DERRIDA, J. [1990]: Mémoires d'aveugles : l'autoportrait et autres ruines, Paris, Réunion des musées nationaux.

Deshoulières, V. [2005] : Métamorphoses de l'idiot, Paris, Klincksieck. DOUBROVSKY, S. [1988] : «Autobiographie/Vérité/Psychanalyse», Autobiographiques : de Corneille à Sartre, Paris, PUF, 61-79.

GRANGE, M.-F. [2008] : L'Autoportrait en cinéma, Rennes, Presses universitaires de Rennes.

JOUHANNAIS, J.-Y [2003] : L'Idiotie: art, vie, politique-méthode, Paris, Éd. Beaux-arts magazine-livres.

LEJEUNE, P. [1975] : Le Pacte autobiographique, Paris, Seuil.

MiCHAUD, P. A. [2000] : "Passage de l'octroi" sur le travesti au cinéma ", La Différence des sexes est-elle visible? Les hommes et les femmes au cinéma, Paris, Cinémathèque française.

MOINE, R. [2002] : Les Genres cinématographiques, Paris, Nathan.

NANCY, J. L. [2000] : Le Regard du portrait, Paris, Galilée.

Parachute [2002] : "Autofictions », no 105, Montréal, Parachute (revue d'art contemporain).

ODIN, R. [1977] : "L'entrée du spectateur dans la fiction», dans J. Aumont et J. L. Leutrat (dir.), Théorie du film, Paris, Éd. Albatros, 198-213 ; [2000]: De la fiction, Bruxelles, De Boeck Université.

Rosset, C. [1977] : Le Réel: Traité de l'idiotie, Paris, Minuit. 